\title{
Thorny Issues of Stakeholder Identification and Prioritization in Requirement Engineering Process
}

\author{
Shariful Islam Majumdar ${ }^{1}$, Md. Saidur Rahman ${ }^{2}$, Md. Mijanur Rahman ${ }^{3}$ \\ 1, 2,3 Department of Software Engineering, Daffodil International University, Dhanmondi, Dhaka, Bangladesh
}

\begin{abstract}
Identifying the stakeholder in requirement engineering process is one of the critical issues. It performs a remarkable part for successful project completion. The software project largely depends on several stakeholders. Stakeholder identification and prioritization is still a challenging part in the software development life cycle. Most of the time, the stakeholders are treated with less importance during the software deployment. Additionally, there is a lack of attempt to think about the right project stakeholder by the development team. In maximum cases, the stakeholder identification technique is performed incorrectly and there is a lack of attempt to mark out them with priority. Besides, there are so many limitations on the existing processes which are used for identifying stakeholders and setting their priority. These limitations pose a negative impact on the development of software project, which should be pointed out by giving deep concern on it. We are aiming to focus on this typical fact, so that we can figure out the actual problem and current work on identifying stakeholders and setting their priority.

Keywords: Stakeholders, Stakeholder Identification, Stakeholder Selection, Stakeholder Prioritization, Stakeholder Value, Software Development
\end{abstract}

\section{Introduction}

Requirement engineering is the most important phase in software development life cycle. Identifying, analyzing, specifying, managing, verifying and validating the system's requirements are the main purpose of requirements engineering. The quality of the software depends on well-documented requirements. Moreover, missing the exact requirements may cause the software failure.

Requirement engineering process is designed with some common series of stages elicitation, analysis, specification, validation, and management [1].In requirements engineering, requirements elicitation is the practice of collecting the requirements of a system from users, customers and other stakeholders. Improving the elicitation process needs to understand and describe the existing methods which are being use for gathering requirements in software developments projects. Improving the requirements elicitation requires us to first understand the stakeholder identification process [2].Besides, the requirements elicitation process must be done in a way where all stakeholders' involvements are ensured.

A stakeholder in an organization is (by definition) any group or individual who can affect or is affected by the achievement of the organization's objectives [3]. Stakeholder can be anyone who has to take action for an organization or who has to be affected by the project. In another term, when a project commences for an organization, people having any kind of link with that project or organization must be called as a stakeholder. Generally, they are the most important part for any kinds of project. It is because, the stakeholder are responsible for running and maintaining the system of an organization.

As far, stakeholders are the key terms in comparison with others in software elicitation process. The stakeholders must be identified by giving some extra concern and the priority must be specified with the key stakeholders. Several approaches have already been done to satisfy the stakeholder identification and prioritization process. But, it is not enough to mitigate the major challenges of stakeholder management process. One probable cause is that this process is mistakenly viewed as a self-evident task in which direct users, clients and the development teams are the only stakeholders [4].Whereas, there are some other internal or external stakeholders who may have important relationship and connection with the project.

There are so many theoretical and practical resources, but this system is limited with proper scope of stakeholder management. The research study has to give more importance on this issue. Therefore, there should be a proper and clean literature review to understand the existing fact of stakeholder identification and prioritization process so that in future an error free model or system can be proposed.

\section{Stakeholder Analysis}

In software requirements engineering stakeholders are defined in various terms. 'System stakeholders are people or organizations who will be affected by the system and who have a direct or indirect influence on the system requirements' [5].In another terms' stakeholder is anyone whose jobs will be altered, who supplies or gains information from it, or whose power or influence within the organization will increase or decrease' [6]. 
However, there is still some approach to be done for this complicated issue. In a software project it is too much important to collect the requirements from the appropriate stakeholder, but in general it is one of the paramount issues of identifying and understanding the ideal stakeholder for the project. This results in the nonexistence of clear and systematic proceedings for identifying them in an efficient way [7]. Stakeholders in general can be classified into four types: primary, secondary, external and extended stakeholders [8].The identification can also be based on the system types, system goals and strategies to fulfill the goals as well as system domain [9].

Stakeholder selection is a simple recursive procedure, starting from an initial contact person, and asking each interviewed person who might be worth speaking to. The procedure terminates when no new names arise or when the new names are found not to be relevant [3].

Distinguishes clients, customer, and other stakeholders including subject matter experts, marketing people, product managers and so on attempts to prioritize them: 'the principal stakeholders are the users, clients, and customers' [10].

It also distinguishes 'users' from clients, suppliers, managers 'who are concerned for the system to succeed' (and by implication other people with that concern), and regulators, and it briefly discusses the role of people in the development organization [11].

Stakeholder prioritization is an important as stakeholder identification. When a project commences, there are many stakeholders who desired to be involved in the process [12]. But, all of them may not be essential for the project or may not concern as a key stakeholder. Whether, prioritization the key stakeholder is treated as a smart movement on the requirements engineering process.'Stakesource 2.0 prioritizes requirements using the stakeholders' rating on the requirements and their influence in the project [13]. A kind of sorting process has to be established where certain aspects of the stakeholders enable them to be on top of the list. The sorting is called prioritization. Interpersonal skills are important to ensure an effective requirement engineering process $[14,15$, $16]$.

\section{Related Work}

During the software development life cycle, the actual requirement is collected from the potential stakeholders for a specific system. There are so many proposals on discovering all stakeholders of a specific system which can be domain-independent, effective, and pragmatic. All of the references emphasis the importance of identifying stakeholders, and although they provide examples, or broad guidance for identifying them, none describes a model or a concrete approach for identifying stakeholders for a specific system. Several approaches have done to satisfy these issues.

A stakeholder in an organization is (by definition) any group or individual who can affect or is affected by the achievement of the organization's objectives. According to the Freeman it has been also said that "A stakeholder is anything influencing or influenced by the firm". But it indicates a wide set of identification of stakeholder. Because, there are so many people involved in an organization may be treat as a stakeholder but, rare of them would have the ability to influence or being influenced by the organization itself [17]. On the other hand, the term stakeholder also means "all those who have a stake in the change being considered, those who stand to gain from it and those who stand to lose" [18]. "Stakeholders are all those claimants inside and outside the firm who has a vested interest in the problem and its solution" and "are the concrete entities that affect and in turn are affected by a policy". They suggested ways of identifying stakeholders including: considering standard demographic groups (age, sex, etc) for relevance; asking people who they consider to be the key stakeholders; and studying accounts of ethnographic fieldwork to discover who seems to have a valid interest [19]. That's why, when a project commencing, it is important to figure out the real stakeholder and make sure about their involvement until the project concluding.

A set of stakeholder which can be refer as 'baseline' stakeholders who can recognize 'supplier' stakeholders and 'client' stakeholders from this 'baseline' stakeholder: the former provides information or supporting tasks to the baseline, and the latter processes or inspects the products of the baseline. Other stakeholders that call interact of satellite with the baseline in a variety of ways. Interaction may involve communicating, reading a set of rules or guidelines, searching for information and so on. Focus on interactions between stakeholders rather than relationships between the system and the stakeholder, because they are easier to follow. Baseline stakeholder is divided into four groups: users, developers, legislators, and decision-makers [3].

Stakeholders in general can be classified into four types: primary, secondary, external and extended stakeholders. Primary stakeholders are vital since the outcomes of the project affect them directly and their interests in the proposed system are high. Missing any primary stakeholders can affect the project development and influence the achievement of the project goals. Primary stakeholders normally include individuals who have the power, authority and responsibility over the resources such as financial. Secondary stakeholders embrace those who are affected by the project outcomes indirectly. They may be the consumers of a product or service. 
Although, they do not participate in project development matters, they monitor the fulfillment of their interests. External stakeholders are not directly a part of the project team but they add values to the project from outside. Extended stakeholders could be anyone who is often helpful in assisting above-mentioned stakeholders to reach their visions [8].

Robertson states that the term stakeholder encompasses sponsors (needed for organizational commitment), consultants (knowledgeable either in technical aspects or the problem domain), and influencers (culture, law, inspectors, competition...etc.). Finally, she extends the term to include users who she defines as one of many consumers. She adopts the term consumer to refer to the many different roles that a person may take and the impact they have on the success of the product, namely that of the buyer and users. People who play the role of buyers are those who decide whether or not to buy the product. Whereas, she identifies users as people who come into direct contact with the product [20].

The center of the theory is success-critical stakeholders' win-win Theory W, which address what values are important and how success is assured for a given software engineering organization. The four supporting theories that it draws upon are dependency theory (Identifying all of the success-critical stakeholders), utility theory (Understanding how the success-critical stakeholders want to win), decision theory (Having the success-critical stakeholders negotiate win-win product and process plans), and control theory (Adaptively controlling progress toward a success-critical stakeholders win-win outcome) [21, 22].

Stakeholders of a system may vary throughout the life cycle. Thus, in order to get a complete set of needs and subsequent requirements, it is important to consider all stages of the life cycle when identifying the stakeholders or classes of stakeholders. Every system has its own stages of life, which typically include stages such as concept, development, production, operations, sustainment, and retirement. For each stage, a list of all stakeholders having an interest in the future system must be identified. The goal is to get every stakeholder's point of view for every stage of the system life in order to consolidate a complete set of stakeholder needs that can be prioritized and transformed into the set of stakeholder requirements as exhaustively as possible [23].

Stakeholder mapping is an important step to understand who your key stakeholders are, where they come from, and what they are looking for in relationship to your business, which can draw from multiple perspectives to determine a key list of stakeholders across the entire stakeholder spectrum. Gather a crossfunctional group of internal participants and identify sources external that may have important knowledge about or perspective on the issues, and reach out to these sources for input as a list of stakeholder. This list will change as the environment around you evolves and as stakeholders they make decisions or change their opinions. It is true that there is no magic list of stakeholder which will not change for any kind of project. The final list will depend on your business, its impacts, and your current engagement objectives-as a result it should not remain static [24].

The key idea to develop a comprehensive understanding is who you stakeholders are, what they care about, and how they relate to the initiative of your project. The idea sounds simple enough, but many big programs either neglect it altogether or limit consideration to the most obvious stakeholder. In fact, due to their scope and complexity, most enterprise-wide programs require a more comprehensive scan to identify the many disparate stakeholders involved and to understand the unique needs and interests of each. In performing initial scan, it's useful to view the stakeholder landscape from multiple dimensions: vertically, horizontally, and from outside the organization.

The vertical scan: The reach of most large-scale projects team extends all the way up to an organization's senior leadership ranks and down to individual end users. Knowing the key players at each level of an organization, and how each relates to the program, is the first step toward crafting an effective outreach strategy.

The horizontal scan: Across an organization, there are likely to be many stakeholders whose roles relate to your planned program in different ways. Each "functional" stakeholder represents a different perspective and type of expertise.

External scan: Often, major sourcing programs will apply all their energy and resources to engaging the internal stakeholder community but will neglect the needs and interests of key external constituents.

Others: such as special interest groups or regulatory bodies, may have significant impact on a program.

A stakeholder mapping exercise like this is useful for identifying stakeholder groups at an aggregate level. But a comprehensive stakeholder analysis must also consider the key individual stakeholders within each group because their buy-in and involvement are needed if the program to be success.

Once individual stakeholders have been identified, a useful exercise to prioritize each based on two criteria: (1) the degree of influence they have on program outcomes and (2) their "attitudes" toward the program, either positive or negative. Individual stakeholders can be groped loosely into the following four categories, with outreach strategies that are unique to each: 
- High-Influence Challengers: Outreach efforts should focus on converting these individuals to champions. Failing that, plan countermeasures that could help to neutralize any actions they might take that could potentially harm or derail the program.

- High-Influence Champions: Proactively leverage the positive energy from these individuals to further program objectives and to build a strong foundation of support.

- Low-Influence Challengers: Maintain awareness of any actions that could potentially harm the program, but put less energy into converting these challengers to champions.

- Low-Influence Champions: Ensure that positive relationships are maintained, but put less energy into further cultivating these champions [25].

At the beginning of any project, the main task is to set clear project definitions that include project goals and system descriptions (type and domain). The project goals specify what the business wants to achieve through the project while the system descriptions define the characteristics of the system to be built. The definitions lead to the recognition of which types of stakeholders (primary, secondary, external and extended) are required. Stakeholders have specific roles. They can therefore be categorized based on the roles that they are playing. Stakeholders may also be assigned to more than one role. Moreover, roles have degrees of importance. Some stakeholders' roles are more influential and significant than the others. These roles have higher chances of being considered in the next stage. In order to determine which groups of stakeholders should be considered, they can be classified into the following classes:

- Mandatory (M) -Stakeholders that must be included or else the success of the system is threatened.

- Optional (O) - Stakeholders that are not necessarily selected. By neglecting their needs does not threaten the success of the system.

- Nice-to-have $(\mathrm{N})$ - Stakeholders that do not influence the system's success if they are not selected.

Due to its importance, primary stakeholders are considered as $M$ regardless the roles that they are playing. Secondary stakeholders may fall under $\mathrm{M}$ or O, depending on their roles' degrees of importance. External stakeholders are mainly $\mathrm{O}$ but they may become $\mathrm{N}$ if their involvement or roles towards the project are insignificant. Lastly, extended stakeholders fall under $\mathrm{N}$ as their contributions are quite minimal [12].

Projects have constraints which hinder project managers to include all possible stakeholders into a project. A kind of sorting process has to be established where certain aspects of the stakeholders enable them to be on top of the list. The sorting is called prioritization. Interpersonal skills are important to ensure an effective RE process $[14,15,16]$. The skills therefore can be used as the final measures to qualify the selected stakeholders as the best possible participants. The skills include negotiation, collaboration and communication (written and oral). These three skills have to be considered holistically, which can be measured through predetermined tests. Some possible prioritization techniques that can be adopted include the ones that are normally employed in prioritizing requirements such as Analytical Hierarchy Process (AHP) [26], Case-Based Ranking [27] and Hierarchical Cumulative Voting [28].

Reviewing the literatures which are based on identifying the stakeholders in requirement engineering, we understood that the sources of favorable requirements depend on wide range of stakeholders. In spite of several ideas to identify the right stakeholders, these techniques are kept down and isolated. It is not immaculate that how elements of project are interrelated between sources of requirements and stakeholders. We are supposed to discover a new model integrating these elements so that their effects on the matter can be seen expressly. On the other hand, there is no appropriate method available to prioritize identified stakeholder based on any value metric. Despite, stakeholder prioritization is another ticklish issue for a successful project, because to elicit the collected requirements of a specific system, it can be conflicted for various approaches encountered from wide range of stakeholders. To get over this type of problems, we have to create a new effective model so that based on that model; a software team can easily prioritize the identified stakeholders regarding a specific system.

\section{Research Methodology}

During studying this paper, normally a technical person or a novice reader regarding software engineering can be pointed out some research questions as like below which are imparted detail. Our aim is to explain the critical phases of a successful software project.

- What are the sources of requirements that need to be considered when identifying stakeholder?

- What elements are required which to be taken into account during stakeholder selection?

- What challenge can be encountered when identifying the stakeholder?

- How to understand that the identified stakeholders are right or not?

- What problems will may face during stakeholder prioritization?

In a large project of a computer system, the sources of requirements may involve a wide range of people. Identifying the sources of requirements is very difficult in real life. Sources may be end user, an interfacing system, or environmental factors [29]. 
To answer the question how to identify the stakeholder, you can analyze the content that is related to desired system. Content analysis was chosen because it helps to answer the questions what and how to the study identifying stakeholder. Content analysis is a systematic, replicable technique for compressing many words of text into fewer categories based on explicit rules of coding [30]. By conducting the content analysis, it may be helped you to identify abstract categories that will assist to select right stakeholder.

Stakeholders in familiar domains can be identified readily enough. The challenges lies in identifying those that exist in poorly documented or poorly understood domains which are to remote software engineers [31]. Some suggest that in such cases product development could include ethnographic fieldwork and other nontraditional qualitative research methods to identify key stakeholders [32]. However, they also state that travel to the target domain may be prohibited or interacting with the stakeholders is problematic because of conflicting views of who constitutes a stakeholder [31].

The key is not to agonize over whether your stakeholder list is "right". By following through the below questions, you will have created a robust, relevant, prioritized stakeholder list-but it will change over time. You should focus on below questions whether that answering list will help you further prepare for right stakeholder engagement activities.

- Is your list focus on relevant stakeholders who are important to your current and future efforts?

- Do you have a good understanding of where stakeholders are coming from, what they may want, whether they would be interested in engaging with our organization, and why?

- Based on your selected stakeholders, can you define a granular level of engagement? Will this list inform tactics, formats, and investment considerations?

- Have you given through to what type of resources (expertise, people, and budget) you need to support your engagement strategy and follow-up activities [24]?

In spite of this process as like a complicated way for someone, answering the following questions you can decide that the selected stakeholders are able or not to fulfill your desired requirements.

\section{Problem Identification}

During literatures review, some problems were encountered to us when identify and prioritize the stakeholders regarding software project. Literatures do not help in identifying right stakeholders for a specific system and do not take any step about the solution how to prioritize the selected stakeholder. We attempt to find out ten major problems those were encountered during the papers reviewing.

- All relevant stakeholders are consulted with less attention.

- Software engineering community does not address how stakeholders are identified in remote regions despite acknowledging that locality and culture typically have an impact on a software product.

- There is a need of systematic approach that could help in identifying and choosing the appropriate stakeholders.

- As stakeholder participation can be enormous approach, it should also embrace prioritization.

- Existing methods to identify and prioritize stakeholders do not scale well to large project.

- Existing stakeholder identification methods require substantial efforts from the requirement engineering when there are many stakeholders.

- The existing prioritization methods fail to appropriately structure the data for stakeholder value. This problem is often compounded by a failure to handle multiple stakeholder view points.

- One of the main problems in requirement engineering is lack of necessary skills of stakeholders to elicit the requirements.

- Stakeholders normally have different concerns, objectives and responsibilities. When multiple stakeholders participate in a discussion, requirements often conflict.

- Maximum time, software engineer groups were utterly confused what are the required elements that need to be run after identifying the stakeholders.

Although the stakeholders play a major rule in a software product, the software engineering community does not find out how to identify and set priority of stakeholders, despite acknowledging that stakeholder is an important part of a successful software project, so less attention to stakeholders may cause for failing down a system.

\section{Conclusion}

Engagement of right stakeholders is immensely important for a large project. Stakeholder is an individual or a group who can influence or be influenced by the success or failure of a project. The systematic literature review provides us with an indispensable way to understand the process, how the stakeholders are being identified in requirements engineering process. Besides, giving concerns on different approaches of stakeholder identification and prioritization process to point out the limitations, which are remarkable for successful project completion. Giving less attention to find out appropriate stakeholders by software community 
can be the cause of project failure. We, therefore, should engage right stakeholder based on sources of requirement for a specific project.

\section{References}

[1] I.Sommerville, and P.Sawyer, Requirements engineering: A good practice guide (John Wiley and Sons, 1992). Nuseibeh, and Easterbook, Requirements engineering: A roadmap, Proceeding of the ACM Conference on Software Engineering, Limerick, Ireland, pp.35-46.

[2] Sharp, Finkelstein \& Galal, Stakeholder identification in the requirements engineering process, Proceedings of 10th International Workshop on Database \& Expert Systems Applications (DEXA), 11/12/99, pp. 387-391.

[3] Carla Pacheco, Ivan Garcia, A systematic literature review of stakeholder identification methods in requirements elicitation, The Journal of Systems and Software, 85, 2012, 2171-2181.

[4] Kotonya, G. and Sommerville, Requirements engineering: process and techniques (John Willey, 1998).

[5] Dix, A., Finlay, J.Abowd, G. and Beale, Human-computer interaction (Prentice-Hall, 1993).

[6] Coughlan J, Lycett M, and Robert D Macredie, Communication issues in requirements elicitation: A content analysis of stakeholder experiences, Information \& Software Technology, 45(8), 2003, 525-537.

[7] J. McManus, A stakeholder perspective within software engineering projects, Proceedings of IEEE International Conference on Engineering Management, Vol. 2 , 2004, pp.880-884.

[8] O. Preiss and A. Wegmann, Stakeholder discovery and classification based on systems science principles, Proceedings of 2nd AsiaPacific Conference on Quality Software, 2001, pp. 194-198.

[9] Robertson, S. \& Robertson, Mastering the requirements process (Addison-Wesley, 1999).

[10] Alexander, I. \& Stevens, Writing better requirements (Addison-Wesley, 2002).

[11] Rozilawati Razali, Fares Anwar, Selecting the right stakeholder for requirement elicitation: A systematic approach, Journal of Theoretical and Applied Information Technology, Vol. 33, No. 2, 30 $0^{\text {th }}$ November 2011.

[12] Soo Ling Lim, Daniela Damian, Anthony Finkelstein, StakeSource2.0: using social networks of stakeholders to identify and prioritise requirements, 33rd International Conference on Software Engineering, 2011, pp.1022-1024.

[13] I. Alexander and S. Robertson, Understanding project sociology by modeling stakeholders, IEEE Software, Vol. 21, No. 1, 2004, pp. 23-27.

[14] J. Price and J. Cybulski, Consensus making in requirements negotiation: The communication perspective, Australasian Journal of Information System, Vol. 13, No. 1, 2005, pp. 209-224.

[15] F. Anwar, R. Razali and K. Ahmad, Achieving effective communication during requirements elicitation - A conceptual framework, Communications in Computer and Information Science, Vol. 181, No. 5, 2011, pp. 600-610.

[16] Freeman RE, Strategic management: A stakeholder approach (Pitman, Boston, 1984).

[17] L. Macaulay, Requirements capture as a cooperative activity, Proceedings of IEEE International Symposium on Requirements Engineering, 1993, pp. 174-181.

[18] Mason R.O., Mitroff I.I., Challenging strategic planning assumptions: Concepts, methods, and techniques (Wiley, 1981)

[19] Robertson, S., Project sociology: Identifying and involving the stakeholders. The Atlantic Systems Guild Ltd, 2000.

[20] B. Boehm, et al., Guidelines for lean model-based (System), Architecting and Software Engineering (Lean MBASE), USC CSSE, 2007.

[21] B. Boehm, J. Lane, Using the incremental commitment model to integrate system acquisition, Systems Engineering and Software Engineering, CrossTalk, 2007.

[22] Stakeholder needs and requirements, http://www.sebokwiki.org/wiki/Stakeholder_Needs and Requirements.

[23] Eric Olson, Jeremy Prepscius, Farid Baddache, Stakeholder mapping, Business for Social Responsibility, November 2011.

[24] Raj Sharma, Supply chain management review: The six principles of stakeholder engagement, www.scmr.com, October 2008.

[25] T.L. Saaty, The analytic hierarchy process (McGraw-Hill, New York, NY, 1980)

[26] P. Avesani, C. Bazzanella, A. Perini, and A. Susi, Facing scalability issues in requirements prioritization with machine learning techniques, proceedings of $13^{\text {th }}$ IEEE International Conference on Requirements Engineering, 2005, pp. 297 - 305.

[27] P. Berander and P. Jonsson, Hierarchical cumulative voting - prioritization of requirements in hierarchies, International Journal of Software Engineering and Knowledge Engineering (IJSEKE), Vol. 16, No. 6, 2006, pp. 819-850.

[28] Nilofar Mulla, Sheetal Girase, A new approach to requirement elicitation on stakeholder recommendation and collaborative filtering, International Journal of Software Engineering and Applications, Vol.3, No.3, May 2012.

[29] R. P. Weber, Basic content analysis (Sage, 1990).

[30] Ban Al-Ani, David Redmiles, Challenge encountered when identifying remote stakeholders, University of California, Irvine, CA 92697-3440 USA.

[31] Foucault, B. E., R., Bell, Techniques for researching and designing global products in an unstable world: A case study, Extended Abstracts on Human Factors in Computing Systems, Vienna, Austria, April 24-29, 2004, 1481-1484. 